\title{
Out from the cold
}

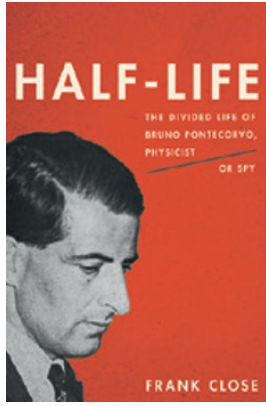

\author{
Half-Life: The \\ Divided Life of \\ Bruno Pontecorvo, \\ Physicist or Spy
}

By Frank Close

BASIC BOOKS: 2015. 400 PP. $€ 20$

3

runo Pontecorvo's defection to the Soviet Union in 1950 is one of the more singular events in the history of Cold War science. A talented physicist who had been involved in wartime nuclear research, his abrupt decision to flee behind the Iron Curtain with his family is shrouded in mystery. Was Pontecorvo a Soviet spy or an idealist looking to escape anti-communist hysteria in the West, consciously choosing to live in Russia?

In Half-Life, Frank Close, a professor of physics at the University of Oxford, sets out to answer this question. He does so by painstakingly bringing together evidence from archives, letters and interviews with surviving family members and scientific colleagues. As one might expect for an unsolved Cold War mystery - especially one involving secrets about the nuclear bomb - others have already investigated and written about Pontecorvo's defection, most notably Simone Turchetti in The Pontecorvo Affair (Univ. Chicago Press, 2012) and Miriam Mafai in Il Lungo Freddo (BUR, 2012). What sets Close's work apart is that, in addition to bringing to light new archival material obtained from the UK intelligence agency MI5, it also describes in detail the context and significance of Pontecorvo's research over the course of his career. The result is, if not quite the solution to the mystery of Pontecorvo's sudden disappearance, certainly an exhaustive analysis of the factors that would have precipitated it.

Pontecorvo was born into a wealthy Jewish family near Pisa, Italy, in 1913. After studying with Enrico Fermi in Rome, where he participated in pioneering experiments with slow neutrons, he won a scholarship in 1936 to work in Paris with Irène and Frédéric JoliotCurie, who had just received the Nobel Prize in Chemistry for their work on radioactive elements. Pontecorvo's time in Paris was eventful. In addition to working on nuclear isomerism, he came into contact with a vibrant and cosmopolitan political scene. Having already embraced the antifascism movement in Italy, this environment was the catalyst for shaping his communist beliefs. Pontecorvo also met his future wife, Marianne Nordblom. War broke out soon after they had their first child, and following the Nazi occupation of Paris they fled to the USA in 1940.

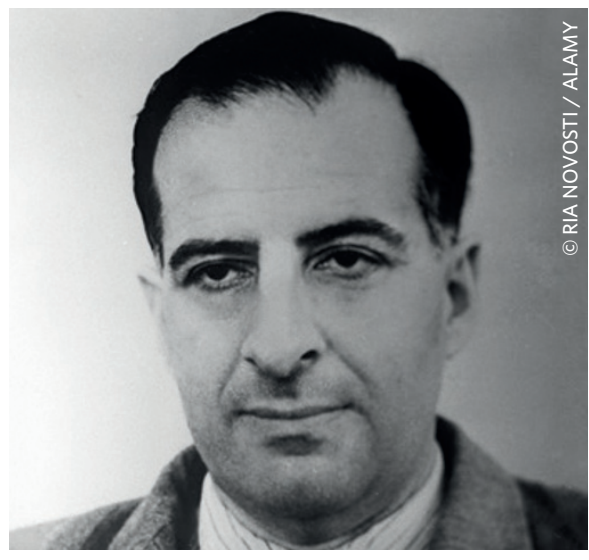

In the USA, Pontecorvo developed the first industrial application of neutrons for geophysical prospecting, by looking at how they interact with different rock formations and working out when there was oil underground. Soon after, in 1943, he was co-opted into the Anglo-Canadian arm of the Manhattan Project by an old Parisian contact, and moved to Chalk River Laboratories to work on the 'pile physics' of nuclear reactors. During this time he had access to classified information that would have been of interest to the Soviets, and Close presents a body of circumstantial evidence suggesting that he might indeed have been leaking this information.

After the war ended in 1945, Pontecorvo remained in Canada and devoted himself to pure science: it is during this period that he proposed, with input from his colleagues at Chalk River, the chlorine-argon method for detecting neutrinos, and suggested that the Sun would be a considerable source of these ghostly particles. He also made pioneering insights, at least on a qualitative level, regarding the existence of the weak force. Close describes these discoveries in detail, and is almost forensic in breaking down precisely which aspects can be credited to whom, but his admiration for Pontecorvo the physicist is clear.

After his stint in Canada, Pontecorvo moved to the UK in 1949, to work for the Atomic Energy Research Establishment in Harwell. And it is at this point that
MI5 started to take a keener interest in his political leanings. After concluding that it was "difficult to regard a man with Pontecorvo's international outlook and family history as reliable" for sensitive nuclear projects, a face-saving solution was engineered, in the form of a professorship at the University of Liverpool. But Pontecorvo never took this appointment: in that fateful summer of 1950, midway through a family holiday in Italy, he abruptly headed to Stockholm and onwards through Finland to the Soviet Union, only to resurface publicly five years later.

What triggered Pontecorvo's sudden flight? There is no smoking gun to prove that he ever passed secrets to the Soviet Union. But Close concludes that the kaleidoscopic body of facts surrounding his defection only falls into place once one accepts the hypothesis that he had been a spy prior to 1950 - any other interpretation requires an unwieldy number of additional explanations, akin to the epicycles invoked by medieval astronomers looking to keep the geocentric model of the Solar System consistent with observation. Moreover, Close has also uncovered a letter from the British embassy in Washington to the Director General of MI5 in London, raising concerns about Pontecorvo's political views stretching back to his days in the USA. Close contends that this would have gone through Kim Philby, the double agent who was stationed in Washington at the time, who would have tipped off Moscow; in turn, the Soviets would have warned Pontecorvo that his cover had been compromised and facilitated his defection. Thus began the second half of his life, as a research scientist in Dubna.

Whereas the book will inevitably attract readers interested in a good story about espionage, Half-Life is also a masterful reappraisal of Pontecorvo's scientific achievements. Although his most famous work is on neutrinos - their co-existence with muons and oscillating nature are two of the more famous insights he made during his time in Dubna - what is most striking is the sheer breadth of Pontecorvo's work over the course of his entire life. In Ugo Amaldi's words: "How many have made great contributions in both theory and experiment? Fermi, Rutherford, Pontecorvo - not many at Nobel level." That he was never awarded the prize may have been the price for his flight to the USSR, suggests Close. He leaves us in no doubt that he would have been a worthy recipient.

REVIEWED BY ANDREA TARONI 